\title{
La teoría de la mente según el simulacionismo científico
}

\author{
Michael Padilla-Mora \\ Programa de Investigación en Neurociencias \\ Programa de Cognición y Lenguaje \\ Universidad de Costa Rica \\ Ce:michaelpadillamora@gmail.com
}

\begin{abstract}
Resumen. Resultados recientes de los trabajos en neurociencias describen algunos puntos fundamentales relacionados con el funcionamiento de la teoría de la mente (TdM) en el homo sapiens sapiens. Algunos hallazgos han suscitado polémica entre los seguidores del simulacionismo y los adeptos de la teoría-teoría, privilegiando especialmente el punto de vista de los primeros. En este artículo se argumenta que, lejos de regresar a una discusión polarizada, se requiere el desarrollo de un constructo de la teoría de la mente (TdM) acorde con el gran cuerpo de resultados de la investigación científica. Ni el simulacionismo ni la teoría-teoría, así como tampoco el modularismo ni una perspectiva híbrida entre esos enfoques parece capaz de dirigir un verdadero progreso. En su lugar, una noción de la TdM humana en términos de nuestra constitución biológica y de los recursos que en esa constitución pueden verse instanciados, podría ser un camino más indicado. La evidencia neurocientífica se sugiere como un buen cimiento para ese proyecto.
\end{abstract}

Palabras clave: teoría de la mente, neuronas espejo, simulacionismo, neurociencia cognitiva, cognición social.

Abstract. Recent results from the work developed in neuroscience seem to describe some fundamental points related to the operation of the theory of mind (ToM) among the Homo sapiens sapiens. The news has provoked controversy between the followers of the "simulationism" and the theory-theory supporters, granting with a special privilege to the view of the formers. Nevertheless, what we need right now, far from a return to a polarized discussion, is the development of a construct on theory of the mind (ToM) in accordance with the great body of the scientific research results. Neither the simulationism, nor the theory-theory, nor the modularism, or a mixed-hybrid perspective seems to be able to lead a true progress. In its place, a more appropriate project can be developed if we adopt a notion about human ToM in terms of our biological constitution and the resources that can be instantiated in that constitution. The evidence from neuroscience is suggested as a good support for that project.

Key Words: Theory of mind, mirror neurons, simulationism, cognitive neuroscience, social cognition. 


\section{Introducción}

Durante muchos siglos, cuestiones que hoy son centrales en el estudio de la teoría de la mente (TdM) humana se mantuvieron al margen de la reflexión para el común de las personas, al igual que para la mayoría de los filósofos y psicólogos.

El asunto escapó de las profundas reflexiones de muchos, posiblemente por tratar una dimensión de la cognición absolutamente cercana a nuestra vivencia cotidiana. Podemos pensar que fueron precisamente la cercanía y la cotidianidad, algunos de los aspectos que mantuvieron el análisis del proceso cognitivo denominado TdM al margen de la reflexión teórica. De cualquier forma, hizo falta mucho tiempo para encontrar términos adecuados para edificar un acercamiento bien estructurado a la manera en que los miembros de nuestra especie atribuyen estados mentales a sus congéneres -e incluso más allá de ellos.

Es alrededor de 1980 cuando el tema de la TdM se afianza sobre la mesa de discusión. Ya en 1977 N. Humphrey se refiere al tema en un congreso y un año más tarde Premack y Woodruff publican su clásico "Does the Chimpanzee have a theory of mind?". De repente parece que todos tienen algo que decir: filósofos, psicólogos desarrollistas, antropólogos, primatólogos, psicólogos sociales, entre otros (Gopnik, 1999). Con el pasar de los años las contribuciones vienen y van, desde y hacia muchos ámbitos distintos, consolidando el área y atribuyéndole excelentes pronósticos en favor de un crecimiento exponencial así de adeptos como de trabajos publicados. Después, sobre la mitad de la misma década de los ochentas, ya es posible identificar el temprano establecimiento de dos acercamientos fundamentales para esta etapa seminal del abordaje a la TdM: las teorías simulacionistas y las teorías estrictamente teóricas. Hoy reconocemos que la enorme riqueza del debate entre ambas aproximaciones alimentó el desarrollo de la investigación por muchos años.

La forma en que cada una de las partes se comprometió a fortalecer su propia posición, al tiempo que dedicaba enormes esfuerzos en la búsqueda, formulación y lanzamiento de contra ejemplos hacia el bando rival, trajo a la luz un cúmulo de temas que difícilmente habrían salido a relucir tan rápido, sin el empuje de ese entusiasmo. Hoy podemos decir que aquella fue una disputa oportuna y necesaria, luego de la cual, sin embargo, ninguna de las partes logró imponerse.

Tras el periodo inicial de la contienda (Perner \& Kuhberger, 2003) quedó claro cómo ambos bandos estaban, al menos parcialmente, en lo correcto, pues la experimentación demostró y reafirmó que los procesos a través de los cuales una y otra parte (simulacionistas y teoricistas), caracterizaban la TdM humana eran todos necesarios (hasta cierto punto) para predecir las acciones y los estados mentales de las demás personas. Así, 
para finales de la década siguiente, ambos acercamientos se abren y se encaminan hacia explicaciones del proceso de cognición superior TdM combinando las que hasta entonces habían sido premisas exclusivas de corrientes divergentes.

Este momento de hibridación teórica coincide, por otra parte, con una coyuntura importante en el ámbito del conocimiento neurocientífico. Tras muchas décadas dedicando sus recursos al estudio de aspectos muy puntuales del funcionamiento del cerebro humano, la neurociencia llega a un punto de madurez tal, que sus investigadores (con el apoyo y la credibilidad de una metodología suficiente, extraordinarios instrumentos de registro y resultados ampliamente verificables), se permiten aventurarse en la exploración de algunos de los procesos más complejos de la cognición humana (Gazzaniga, 1999). La neurociencia se suma a la ciencia cognoscitiva en su afán de esclarecer los procesos en ejercicio durante las tareas de TdM. Veamos.

\section{Teoría de la Mente: la aproximación simulacionista}

Actualmente tenemos a nuestra disposición una inmensa cantidad de caracterizaciones distintas para la TdM. Buena parte de las distintas acepciones que vienen siendo formuladas responden a la naturaleza del acercamiento desde el cual trabaja cada grupo de investigadores. En muchos casos predominan sesgos de áreas específicas, e incluso otras veces se puede identificar la relativa actualidad (o vigencia) de las propuestas en relación con el llamado a la transdisciplinariedad que se lleva a cabo desde el contenido de sus enunciados.

En principio, por ejemplo, algunos mantuvieron que la TdM se trataba de "la competencia para atribuirle al otro, todo un paquete de estados intencionales" (Dennet, 1978, p. 569); luego, otros la especificaron como "un sistema para inferir los estados mentales del otro, a partir de su comportamiento" (Baron-Cohen, 1997, p. 51). Algunos más sostienen que se trata de "la capacidad de interpretar, predecir y explicar el comportamiento de otros, en los términos de sus estados mentales subyacentes" (Dominey, 2005, p. 3); y aún otros tantos la delinean como "la arquitectura mental que permite construir los procesos cognitivos superiores asociados con la proyección de otras mentes y sus contenidos en relación con la propia" (Arce, 2005, p. 54); o bien simplemente como "aquello que nos permite atribuir estados mentales a los demás” (Adolphs, 2003, p. 171). En todo caso, la dimensión teórica no fue siempre tan diversa.

De hecho, para el momento en que Premack y Woodruff (1978) lanzaron su pregunta básica, podían asumirse a lo sumo dos posiciones. Se podía buscar respuesta en una capacidad similar a nuestra habilidad para 
'tomar-perspectiva' pero en el chimpancé, tal y como venía siendo trabajada entonces por la psicología social (Ruby \& Decety, 2003). Por otro lado, se podía hablar de la cuestión como un fenómeno adscrito a un cuerpo de conocimiento general implícito (folk psychology) siempre dispuesto para los individuos de cualquier grupo o cultura (Gordon, 1999). Ambas alternativas parecían insuficientes incluso para responder a la pregunta "¿tienen los chimpancés teoría de la mente?” (Premack \& Woodruff, 1978). Sin embargo, los acercamientos relacionados con cuerpos teóricos como la folk psychology (o naive psychology) predominaron al abordar los casos de primates humanos y no humanos. Luego, las discusiones germinaron respecto de la forma en que tal conocimiento teórico podía ser manipulado en miras de ciertos objetivos, contenidos y reglas generales. Ese fue el panorama hasta que se manifestó el movimiento de las aproximaciones simulacionistas.

El simulacionismo operó como una corriente reaccionaria (Gordon, 1999) frente a los enfoques que se centraron en el posible procesamiento teórico llevado a cabo durante las tareas de TdM (tanto por simios de bosque como por simios de sabana). Primero impulsada por Gordon (1996) y Heal (1996), esta perspectiva de la teoría de la mente logró establecerse como una posible alternativa para el funcionamiento de la TdM, gracias al apoyo de Paul Harris, Alvin Goldman, Stephen Stich y Shaun Nichols entre otros (Carruthers \& Smith, 1996; MIT, 1999).

El simulacionismo no necesita saber de acumulados de conocimiento compartido, ni de reglas o leyes psicológicas (Heal, 1996). En su lugar se vale de los recursos propios de la mente del sujeto como habilitadores de un ejercicio de simulación que sería la base de toda posible predicción e inferencia acerca de los estados mentales de los demás. De acuerdo con la perspectiva simulacionista, cuando una persona se da a la tarea de atribuirle estados mentales a otra, en cualquier situación determinada, utiliza como heurística particular lo que se ha llamado "un ponerse a sí mismo en el lugar del otro" (Gordon, 1996, p. 14). Es decir, que cuando una persona (A) emprende la tarea de inferir los estados mentales que pueden estar pasando por la cabeza de otra persona (B) en una situación cualquiera $(\mathrm{X})$, esa misma persona (A) utilizaría su imaginación (entre otros de sus recursos mentales) para simular que es ella misma la que se encuentra en la situación particular $(\mathrm{X})$ en que se encuentra la otra persona (B). Así, tras reconocer cuales serían sus propios estados mentales si se encontrase en la situación particular de su congénere, la primera persona (A) terminaría atribuyéndole a la segunda (B) esos mismos estados mentales por analogía. Dicho de otra forma, yo podría inferir que tú tienes determinados estados mentales cuando te encuentras en determinada situación particular: primero, imaginándome a mí mismo en la situación particular en la que tú estás, y segundo, atribuyéndote a ti los estados mentales que pasaron por mi cabeza mientras simulaba estar en tu lugar. 
Resulta obvio que todo el ejercicio de la simulación parte en mayor o menor grado de premisas de similaridad y paridad (Heal, 1996) entre los organismos que se simulan mutuamente, así como en los procesos de cognición que puede efectuar cada uno de ellos por separado. Es gracias a la naturaleza intrínsecamente similar (Heal, 1996, p. 76) de uno y otro individuo, que podemos confiar en la posibilidad de establecer correlatos realmente paralelos entre las dinámicas del pensamiento de ambos por medio de la simulación. La experiencia del organismo simulador deja de ser teórica y puede verse en cambio como una vivencia emocional o afectiva, por medio de la cual es viable captar la intencionalidad que albergan otros miembros de nuestra sociedad. Precisamente por enfatizar la primacía de lo emotivo y el sentir vivenciado, este tipo de enfoque se considera una aproximación "caliente" de la TdM, subrayando la diferencia con la posición que persistía en la relevancia de "frías" estructuras teóricas y su forma de uso como la verdadera explicación de este proceso de cognición superior. Gordon (1999) expresó que el elemento emocional, sumado al nivel de automatismo y a la integración de las constantes del grupo social, hacían del simulacionismo una alternativa evolutivamente más plausible que cualquier otra aproximación teoricista, en tanto parecía siempre más económica y directa.

Tomar en cuenta las constantes del grupo social era evidente, pues ¿de qué otro modo podía el sujeto advertir con certeza los estados mentales de otra persona en situaciones diversas, sino reconociendo los elementos integrados en la simulación como variables en función de un contexto? De igual forma, el automatismo podía llegar a tomar dimensiones colosales, cuando por ejemplo, se introducían las interpretaciones de respuestas fisiológicas como indicadores valiosos durante el proceso de simulación.

Para los simulacionistas, la percepción del gesto de un individuo podía convertirse en un factor clave para el esclarecimiento de su estado mental (Weiskopf, 2002). De ello debe entenderse que como parte del proceso de la simulación, una persona puede también hacer uso de su capacidad de copiar los patrones de inervación muscular del otro, para obtener una idea más precisa de lo que experimenta su congénere. Es decir, que el sujeto aprovecha su capacidad para imitar la inervación muscular del rostro de enojo de otra persona, en su propósito de precisar el detalle de la emoción que padece esa otra persona. Según Gordon (1996), cuando uno lleva a cabo la mueca que observó en otro individuo (o bien la imagina) se manifiesta una respuesta fisiológica que brinda información importante acerca de los estados mentales del individuo simulado. Los teóricos del 'simulacionismo' suponían que éstos y muchísimos procesos más implicados en la simulación y dedicados a la interpretación de la información percibida, debían estar ocurriendo en el cerebro de manera encubierta, sin alcanzar el nivel de la conciencia (Carruthers \& Smith, 1996). Contenidos de carácter muy distinto eran todos procesados a través del proceso de simulación y no escaparon ni 
siquiera algunos de los tipos más difíciles como el contenido no explotado (Cummings, 2002) o el no-contenido (Heal, 1996).

Otras fases del procesamiento igualmente importantes para la atribución de estados mentales desde la perspectiva simulacionista, fueron debatidas en una atmósfera de mayor incertidumbre. Se trata de los procesos que debían atenderse, ya no en la interpretación o decodificación de las fuentes de información, sino más bien en la predicción a partir de lo vivenciado por simulación. Gordon (1996) apuntó a rutinas ascendentes que en el mejor de los casos recordaban los más débiles ejercicios de Wittgenstein en sus "Investigaciones Filosóficas" (Wittgenstein, 1936), aunque por supuesto, no contaron nunca con una extendida aceptación. En cambio, la propuesta que consiguió traducirse en referencia obligada del simulacionismo fue la del procesamiento 'off-line' de Stich, Nichols, Leslie y Klein (1996). En ella, los autores enunciaban que la predicción acertada de los estados mentales de otra persona, se llevaba a cabo utilizando la misma maquinaria cognitiva que se utilizaba normalmente en el día a día y en las distintas situaciones cotidianas, con la diferencia de que ahora esta maquinaria era aplicada sobre un input autoevocado que era estructurado en las primeras fases del proceso de simulación. La dinámica del proceso se visualizó como una función de módulos cognitivos donde el percepto normal (real) era suplantado por información almacenada en la memoria, con mucha anterioridad o casi simultáneamente respecto al momento de su recuperación, la cual luego era manipulada según el propósito del sujeto simulador, y sobre la cual se corría el programa de cognición normal, como si se tratara de un 'input' que realmente estaba siendo percibido (Stich, et al., 1996).

Basado en lo anterior Weiskof (2002) describe el simulacionismo como un enfoque de la TdM básicamente integrado por dos componentes: el componente interpretativo y el componente predictivo. El primero de ellos se refiere a la etapa durante la cual se adscriben estados mentales a las demás personas, modelando internamente sus economías psicológicas particulares. A su vez, éste componente se postula como divido en dos estadios: i) la generación de un escenario imaginario y ii) la generación de estados mentales atribuidos sobre la base del escenario formulado. El segundo momento refiere al estadio que se ocupa de la predicción del comportamiento que deberían exhibir las entidades simuladas según los estados mentales previamente atribuidos.

\section{El enfoque neurocientifico}

Hacia la segunda mitad de los años ochentas, muchos investigadores dedicados al estudio del cerebro dirigen su trabajo al esclarecimiento de los estímulos específicos que disparan determinadas neuronas en distintas áreas 
del encéfalo. Las lecturas de las tasas de disparo de las neuronas individuales no se llevan a cabo en homo sapiens sapiens debido a restricciones éticas ligadas a las técnicas utilizadas. En su lugar, otras especies son llevadas al laboratorio esperando que su estudio aporte significativamente a la comprensión de la organización del procesamiento cerebral.

Rizzolatti, Camarda, Fogassi, Gentilucci, Luppino y Matelli (1988) un grupo del Instituto de Fisiología Humana de Parma, trabajan con la parte rostral inferior del área 6 de los macacos, logrando identificar neuronas cuyo disparo se relaciona, no con varios movimientos aislados por parte del individuo, sino con acciones particulares con sus manos o con su boca. Significa que hay neuronas particulares que se activan cuando el animal rasga o toma alguna cosa con la mano, sostiene algún objeto con su boca, etc. De nuevo, la célula responde específicamente cuando el animal lleva a cabo una acción determinada y no solamente algunos de los movimientos separados que la conforman. Gentilucci, Fogassi, Luppino, Matelli, Camarda \& Rizzolatti (1988) trabajan sobre el área 6 inferior, aclarando que la organización de la citoarquitectura en dicha área, se presenta de una manera somatotópica (Gentilucci, et al., 1988), donde la parte dorsal del área representa las manos, la parte ventral representa la boca, etc.

Un hito en la investigación tiene lugar en 1992, cuando se descubren neuronas -después llamadas neuronas espejo (Di Pellegrino, Fadiga, Gallese \& Rizzolatti, 1992)- que no sólo responden cuando el sujeto lleva a cabo una acción determinada (p.e. agarrar un objeto), sino que también responden cuando el sujeto observa a otro sujeto (de su misma especie o de una diferente) realizar la misma acción. En el laboratorio y en uno de los experimentos determinantes, la misma neurona de un macaco se disparó cuando él agarraba una pasa con sus dedos (pulgar y meñique) y de igual forma cuando él observaba al experimentador agarrar una pasa de la misma manera. A "grosso modo", cuando el organismo "A" observaba al organismo "B" realizar una acción "X", cierta fracción de su sistema nervioso central (SNC) se disparaba tal y como si él mismo (el organismo "A") se encontrase realizando la acción observada. Si el macaco observaba a otro macaco aprehender un objeto con su mano de una manera determinada, se disparaban en su cerebro las mismas neuronas que se activaban cuando él mismo aprehendía algún objeto en igual forma. Si la aprehensión observada se llevaba a cabo con el pie, las propias neuronas correspondientes a la representación del mismo movimiento se disparaban en la correspondiente área somatotópica de la parte rostral del área 6 inferior.

¿Era esto el substrato material de los procesos simulacionistas? ¿Simulaba aquel macaco el comportamiento observable de otros organismos? Aunque aquellos aspectos no se encontraban dentro del marco teórico de los investigadores, una vez que los resultados fueron publicados, otros establecieron el vínculo instantáneamente. Tal como se anotó, para entonces 
la neurociencia iniciaba una apertura hacia el estudio de problemas complejos de la cognición humana, por lo que la publicación de los resultados fue muy oportuna.

Altamente representativa de la polémica inminente fue la pronta publicación del trabajo de Goldman y Gallese (s.f). Así, no parecía posible que el debate consiguiera sostenerse por mucho tiempo si la evidencia implicada se limitaba al mono macaco. De ahí que la acumulación de nuevos resultados experimentales relacionados con el sistema de neuronas espejo (SNE), pero ahora dedicados al abordaje del cerebro de sujetos humanos, realizados por el grupo de Parma, lograra despejar la polémica. A partir de aquí, cabe asumir algunas de las perspectivas y argumentos que han pretendido vincular dos temas centrales de este artículo: neurociencia y simulacionismo. En esta búsqueda hacia los términos apropiados de la simulación implicada durante la TdM en homo sapiens sapiens, cabe valerse de la separación que hiciera Weiskopf (2002) del simulacionismo en dos componentes fundamentales para organizar el recorrido a través de la evidencia neurocientífica.

\section{Neurociencia del simulacionismo: ¿los términos del componente interpretativo?}

El simulacionismo reunió una intrincada dinámica de procesos en su explicación de la TdM. La experiencia de la simulación como "una transición entre estados mentales" cuyos contenidos concernían a su vez otros estados mentales (Perner, 1996, p. 94), vislumbraba una complejidad tan grande que apenas era posible esperar entenderla en su totalidad alguna vez. Sin embargo, el resultado de la investigación experimental de los últimos años sobrepasa cualquier expectativa anterior respecto a la complejidad de nuestros procesos de cognición superior en general. El grupo de Parma, por ejemplo, una vez dedicado a la localización y caracterización del SNE en sujetos humanos, encuentra algunos fenómenos realmente interesantes y posibilita la obtención de evidencia sobre recursos explotables en una situación de simulación.

Una de las limitaciones en la aplicación de técnicas de registro, en el descubrimiento de las neuronas espejo en los macacos, fue utilizar la lectura de la neurona individual por medio de electrodo. La aplicación de este procedimiento en seres humanos quedaba estrictamente prohibida debido al grado extremo de invasión. De ahí que se intentan otras hipótesis y procedimientos. En particular Fadiga, Fogassi, Pavesi \& Rizzolatti (1995), en busca del SNE en el homo sapiens sapiens, se plantean que, si en un ser humano las neuronas espejo se disparan de la misma forma que en el macaco, es decir, cuando el sujeto, por ejemplo, 'agarra una roca' y cuando el sujeto observa a otro 'agarrar una roca', entonces, en el momento en que un sujeto 
observa una acción debería manifestarse en él algún tipo de respuesta, tal cómo si él mismo fuese el que estuviera realizando la acción, esto por supuesto, debido a la actividad de aquellas neuronas especializadas.

Con la anterior hipótesis en mente, los investigadores hacen que sujetos experimentales presencien la ejecución de ciertos movimientos corporales e intentan identificar algún tipo de respuesta en sus músculos, utilizando para ello un registro de potencial motor evocado. Sus resultados confirman que en los sujetos que observan la ejecución de algunos movimientos se presenta un pequeño incremento de potencial en los músculos correspondientes, como si hubieran sido ellos mismos quienes ejecutaron la acción. Esta respuesta es evaluada como consecuencia de la actividad de neuronas espejo en la propia zona sensoriomotora de los observadores, quienes aparentemente emitían la respuesta de manera automática (Fadiga et al., 1995).

Además, como resultado colateral de este estudio, en el que también se utilizó estimulación magnética transcraneal, se advierte que en el caso de bomo sapiens sapiens el funcionamiento del $\mathrm{SNE}$ se diferencia en aspectos fundamentales del sistema descrito en los macacos. Mientras en el caso del macaco las neuronas espejo sólo respondían cuando el organismo realizaba una acción determinada, y no cualquiera de sus movimientos componentes por separado, en homo sapiens sapiens las neuronas respondieron de la misma forma cuando el sujeto fue expuesto a acciones específicas y a movimientos aislados de ésas mismas acciones. Es sorprendente la posibilidad de que esto significase una especialización importante en el caso del SNE propio de nuestra especie (Rizzolatti \& Craighero, 2004), así como sorprendía el lugar en el que nos situaba ése rasgo particular, justo como organismos altamente sensibles a la simulación 'encarnada' (Gallese \& Lakoff, 2005) del comportamiento de nuestros congéneres.

Recientemente, se han recogido indicios acerca de un mecanismo a nivel de la espina dorsal, cuya función parece ser la inhibición de esas constantes respuestas motoras automáticas (¿acaso simulacionistas?) que efectuamos frente al comportamiento de las demás personas (Baldissera, Cavallari, Craighero \& Fadiga, 2001). Proporcional al grado de excitación que produce el SNE, este sistema inhibitorio regula el impulso motor y mantiene los niveles mínimos registrados en los experimentos de laboratorio. De allí que incluso la alteración de este sistema inhibitorio haya sido propuesta como causa de la condición de imitación patológica, en la cual los sujetos imitan compulsivamente y sin control, toda postura o gesto de quienes se encuentren a su alrededor (Jeannerod, en prensa).

Las conclusiones de estos trabajos debatían abiertamente sobre la manera en que aparentemente imitamos el comportamiento de los demás en la vida diaria, copiamos sus acciones o reflejamos (del vocablo inglés mirroring) su comportamiento observable de manera automática. Como ya se 
dijo, el registro principal de estas investigaciones se efectuó sobre los propios músculos del cuerpo.

A partir de cada nuevo resultado se estructuraron nuevas preguntas. Si esto era lo que estaba pasando en el exterior ¿qué podía ocurrir en dimensiones más profundas? Tras el trabajo conjunto de un grupo de investigadores franco-italianos en el año 2003 obtuvieron respuestas igualmente sorprendentes.

Esta vez se intentó responder a la pregunta ¿qué mecanismo subyace nuestra capacidad para entender las emociones de los demás? Siguiendo la pista del recién identificado SNE, el equipo de especialistas se cuestionó acerca de la posibilidad de que la comprensión de las emociones, al igual que el reconocimiento de la acción a través de las neuronas espejo, implicase la excitación de las mismas áreas cerebrales activas en el sujeto al momento de experimentar la acción percibida (Wicker, Keysers, Plailly, Royet, Gallese \& Rizzolatti, 2003).

Para su registro utilizaron las imágenes obtenidas por resonancia magnética funcional y sus resultados confirman que, entre otras cosas, cuando un sujeto experimenta disgusto (p.e., tras oler una sustancia pestilente presentada por el experimentador), su cerebro manifiesta activación en las mismas áreas involucradas en la observación de otros sujetos experimentando la misma sensación. Los investigadores estimularon a sus sujetos experimentales con escenas (filmadas con videocámara) en las que se podían observar las expresiones de personas experimentando disgusto (de nuevo, tras oler una sustancia desagradable) y como resultado, se activaron en los sujetos observadores las mismas áreas activas cuando ellos mismos percibieron el hedor. Wicker et al. (2003, p. 657) afirman que "la observación de 'disgusto' automáticamente activa los sustratos neurales utilizados selectivamente durante el procesamiento de la propia experimentación del disgusto (...) los resultados demuestran que observar una expresión facial de disgusto, automáticamente activa la propia representación neural de disgusto".

Este hallazgo vuelve a llamar la atención acerca de la posibilidad de atender al fenómeno descubierto, como otro de tantos recursos verdaderamente explotables durante el estadio interpretativo implicado en el proceso de atribuirle estados mentales a otra persona. El componente interpretativo de la simulación destaca debido a que contiene varios procedimientos básicos de la experiencia simulacionista.

Con lo señalado hasta esta parte pueden introducirse nuevos elementos para contrastar viejas premisas simulacionistas como "ponernos en el lugar del otro", "dejar que los mecanismos encubiertos realicen su trabajo", "reproducir en nosotros mismos circunstancias perceptuales e intraperceptuales similares a las del otro" y muchas otras. Sin embargo, el componente interpretativo implica mucho más. Tendremos que hablar 
también, al menos, del proceso de imaginería creador de nuevos escenarios y de las pistas invaluables tras la copia de las inervaciones musculares.

Primero cabe subrayar que las mismas respuestas del SNE antes tratadas, p.e., al presenciar a un sujeto realizando una acción, fueron verificadas en el laboratorio también cuando los sujetos se daban a la tarea de imaginar esas mismas acciones. Como si el sujeto estuviera ejecutando la acción o se encontrara presenciándola realmente, su SNC y su sistema muscular, mostraron evidencia de la replicación de las acciones que eran imaginadas (Rizzolatti, 2004). Comprobar que a partir de acciones imaginadas un sujeto podía acercarse en determinado sentido a la vivencia de la situación evocada ha contribuido a las hipótesis del simulacionismo.

La exploración del procesamiento cerebral durante tareas de imaginería mental que implicaban objetos pequeños, elementos más complicados y hasta escenarios muy complejos, encontró un nuevo espacio de discusión al verificarse que en las exploraciones imaginarias de configuraciones visoespaciales se presentaba un 'isomorfismo' asombroso entre el estado del cerebro mientras imaginaba un objeto o escenario y el estado del mismo órgano cuando efectivamente se atendía el objeto determinado (ahora frente a uno) o se observaba el escenario desde el interior (Cocude, Mellet, \& Michel, 1999).

En la mayoría de los casos, cuando un investigador proporcionaba a un sujeto, p.e., una pelota roja y minutos después se la pedía de vuelta para finalmente registrar su activación cerebral durante la tarea de imaginar aquella misma pelota, comúnmente se corroboraba que en el cerebro se encontraban activas las mismas áreas que se ocupaban de registrar la pelota cuando efectivamente se tenía al alcance. Dentro de las regiones cerebrales activas durante la tarea de imaginería se detectó incluso el área encargada de procesar el color rojo, entre otras (Cocude et al., 1999).

En este punto es pertinente referirse al fenómeno o sub-procedimiento mejor estudiado, en el contexto del simulacionismo temprano, a saber, la interpretación de la inervación gestual.

Gazzaniga (2005) ha puesto en evidencia que al introducirnos en una conversación con personas extrañas, poco después se comienza a imitar los gestos y modos de los interlocutores. De la misma manera es posible llegar incluso a adoptar posturas similares a las de las personas que observamos en las fotografías de revistas o en el periódico (Jackson, Meltzoff \& Decety, 2004). Los simulacionistas aseguraban que la sola producción de una expresión alegre, como un rostro sonriente, podía cambiar significativamente el estado de ánimo (Gordon, 1996). A esto cabe agregar que desde la percepción de un rostro pueden moldearse no solamente los músculos faciales, sino también determinadas estructuras internas del SNC como el lóbulo parietal inferior o el área sensoriomotora (Rizzolatti \& Craighero, 2004). La viveza de la imagen de un rostro de dolor traduce una experiencia 
muy cercana a ese mismo sentir, activando en nuestro encéfalo las zonas de dolor específicas correspondientes al tipo de sufrimiento que recoge la imagen (Jackson et al., 2004). Se reconoce por tanto que, aún cuando no seamos capaces de percibirlo, los rostros de nuestros interlocutores -en grados mínimos- imitan nuestros propios rostros en tiempo real y a lo largo de casi toda conversación convencional (Gazzaniga, 2005). Estos son algunos de los recursos humanos disponibles para la interacción en el contexto social.

\section{Neurociencia del simulacionismo:} ¿los términos del componente predictivo?

Con los trabajos de Gallese, Fadiga, Fogassi \& Rizzolatti (1996) y los de Rizzolatti, Fogassi \& Galles (2000) se acepta que, por medio de un sistema neuronal de asociación, el observador -durante la observación- es puesto en el mismo lugar 'interno' en el que estaría si ejecutara la acción realmente.

Después de este hallazgo, pocos trabajos hasta ahora han tratado la cuestión de cómo es posible predecir acciones a partir del comportamiento observable. Es sabido que un individuo lleva o llevará a cabo ciertas acciones, a pesar de no poder observarlas directamente. En efecto, "aún cuando el objeto 'blanco de la acción' está fuera de nuestro alcance visual, podemos entender qué acciones llevan a cabo los sujetos" (Umiltá, et al., 2001, p. 155).

En el laboratorio, Umiltá y sus colaboradores (2001), buscando esclarecer los mecanismos detrás de aquella capacidad, se mostró a monos macacos un conjunto de escenas en las que se observaba la mano de uno de los experimentadores aprehendiendo un objeto. Sus neuronas espejo, justo como se esperaba, respondieron tal como si el propio macaco realizara la acción. Después, en una segunda condición experimental, nuevamente se mostró a los macacos la misma escena, pero ahora alterada a través de un ordenador. En la nueva escena resultaba imposible visualizar el final de la acción, es decir, el momento en que la mano entraba en contacto con el objeto. A esta nueva situación, las neuronas espejo de los macacos respondieron exactamente igual que a la condición original (Umiltá, et al. 2001). La información previamente procesada como parte del estímulo parecía ser suficiente para que el macaco construyera el resto a partir de ella, y comprendiera la acción que habría de llevarse a cabo. Lo anterior fue un hallazgo novedoso pues hasta ese momento los disparos de las neuronas espejo sólo se habían registrado cuando el macaco era expuesto a una situación transitiva completa, incluyendo la mano operando directamente sobre el objeto (Rizzolatti \& Arbib, 1998).

Por otro lado, dentro de la corriente simulacionista tradicional, las explicaciones que se ocuparon del problema de la predicción fueron, 
resultado de trabajos como los de Stich, et al. (1996) y similares. El funcionamiento off-line del simulacionismo, el cual anteriormente fue caracterizado como una función de módulos cognitivos, donde el percepto normal era suplantado por información almacenada, sobre la cual se ejecutaba el programa de cognición normal, se encargó del componente predictivo. Algunas evidencias neurocientíficas apuntaban, si no al funcionamiento off-line propiamente, sí a un procesamiento parecido como el mecanismo subyacente al interior del cerebro humano. Las áreas cerebrales, como los módulos en el argumento off-line, operan con casi exacta normalidad sobre el input percibido y sobre el figurado.

\section{Más allá del grupo de Parma}

En las dos secciones anteriores se han revisado, de manera sintetizada, varios argumentos comunes en la discusión actual entre el simulacionismo y la neurociencia. Especialmente en enfoques vigentes y en auge como el de Victorio Gallese, el tipo de "vínculo intersujeto" al que se ha hecho referencia. Este es caracteterizado, sobre cualquier otra clase de explicación, como la raíz de la cognición social (Gallese, 2001; Gallese, Keysers \& Rizzolatti, 2004; Gallese \& Goldman, 1998).

Más allá del grupo de Parma, críticas y evidencia también han sido expuestas como contra-argumentos hacia la posición de Rizzolatti y sus colaboradores (Riva, 2006). Lo que es más, de la misma forma en que hemos procedido hasta esta parte, podríamos ensamblar simultáneamente una cadena de argumentos -también asentados en la neurociencia- que socave la plausibilidad del 'simulacionismo' como procedimiento básico de nuestra TdM.

Los argumentos como los de Rizzolatti y colaboradores, han sido criticados desde otras explicaciones de la neurociencia (Riva, 2006). Por ejemplo, puede considerarse la importancia que tiene para el simulacionismo el "ponerse en el lugar del otro" e "intentar ver las cosas desde su perspectiva", pero ahora ofreciendo para el contraste algunos experimentos realizados con personas diagnósticadas con autismo. Algunas de estas personas con profundas deficiencias en su teoría de la mente (TdM), pueden llevar a cabo las tareas incluidas en los ejercicios psicológicos de 'toma de perspectiva' sin problema alguno (Hobson, 1984). Esto reduce significativamente la relevancia de 'asumir la perspectiva de otra persona' en relación con la capacidad para atribuirle estados mentales.

En la misma línea podría sostenerse que tampoco la capacidad para realizar grandes ejercicios de imaginería viso-espacial -piedra angular del simulacionismo- debería asumirse como un procedimiento necesario dentro de los procesos de la TdM. Aún no puede negarse la posibilidad de verificar, trabajando con personas afectadas por lesiones cerebrales, que limiten su 
capacidad para generar imaginería, que su déficit (aunque discapacitante en muchos otros sentidos) no represente mayor problema a la hora de procesar la atribución de estados mentales (Weiskopf, 2002). Se hace necesario entonces más trabajo experimental para esclarecer este punto.

Mucho menos prudente puede llegar a parecer una adhesión al simulacionismo cimentada en la verificación de estados isomorfos en el SNC durante la observación y producción de acciones, si se yuxtaponen esos resultados contra otros registrados en tareas experimentales aún más cercanas al verdadero ejercicio diario de la TdM. Cuando Ruby y Decety (2003) comparan la actividad cerebral de individuos que emiten su propia opinión acerca de un tema determinado, con la actividad cerebral cuando los mismos individuos intentan expresar la opinión que podría tener otra persona, lejos de subrayar la activación de los mismos circuitos neuronales, más bien enfatizan la activación específica del giro postcentral para la perspectiva de primera persona y la de otras áreas como la zona superior del lóbulo temporal izquierdo para la perspectiva de la tercera persona (Ruby \& Decety, 2003).

También, en el trabajo de Riva (2006) puede encontrarse una muy puntual enumeración de críticas hacia el trabajo del grupo de Parma. Sobresalen allí los comentarios de Jacob y Jeannerod: "la descarga [de una neurona espejo] no es para nada un proceso cognitivo social" (Riva, 2006, p. 61). Tanto Jacob como Jeannerod (como se cita en Riva, 2006) enfatizan que desde el principio, en el laboratorio, el primate A (cuyo circuito neuronal es disparado) no se encuentra implicado en ninguna clase de relación social con el primate $\mathrm{B}$ (quién efectúa el movimiento que dispara el circuito). Para estos autores no puede decirse - a menos que sea en un sentido muy débil- que ambos primates hayan establecido un proceso de cognición social compartido, ni tampoco un proceso intencional, y ni siquiera uno en calidad no-verbal.

Además, Riva (2006) se vale de la estratificación de niveles de entendimiento social que realiza Tomasello (1999), para manifestar una posición más moderada que la del grupo de Parma respecto a la función del SNE. En efecto, al establecer niveles ascendentes de entendimiento social, Tomasello (1999) enuncia los siguientes: a) la capacidad para predecir el comportamiento a partir de las conductas observables; b) la capacidad de entender que los otros individuos dirigen su atención y comportamiento de acuerdo con sus propósitos; c) la capacidad para entender que las creencias de los agentes pueden diferir de aquellas que pueden inferirse por su comportamiento. Partiendo de ese esquema, Riva (2006) argumenta que, en el mejor de los casos, el papel de las neuronas espejo jugaría un rol crítico solo en las dinámicas implicadas dentro del primer nivel. 


\section{La TdM en el contexto del cuerpo}

La contribución fundamental del trabajo neurocientífico al estudio del proceso cognitivo que se denomina "teoría de la mente" (TdM), no se centra únicamente en la descripción del procesamiento neuronal en la parte rostral inferior del área 6 , ni en el hallazgo de circuitos neurales que responden a la observación de acciones transitivas. El verdadero impacto de la evidencia debe ser visto, en su conjunto, como el descubrimiento de un estilo de funcionamiento cognitivo generalizado en Homo sapiens sapiens.

Como se ha mostrado, el estilo de procesamiento en "espejo" ha venido siendo reconocido en nuestra especie, como un modo funcional implicado en cada vez más procesos cognitivos. Primero advertido en el reconocimiento de las acciones, luego en el reconocimiento de emociones. Posteriormente en el ejercicio de la imaginación, en la atribución de dolor y más recientemente en el entendimiento del contacto físico (Rizzolatti \& Craighero, 2004; Wicker et al., 2004) (Colude, Mellet \& Michel, 1999; Gallese, 2006; Rizzolatti \& Craighero, 2004; Wicker, Keysers, Plailly, Royet, Gallese \& Rizzolati, 2004). El procesamiento en “espejo" entendido como la transducción de las acciones observadas a una modalidad cerebral sensoriomotriz y experienciada, se sugiere como un recurso básico en nuestra implementación cotidiana de la TdM.

Asimismo, resulta fundamental que se manifiesten explícitamente algunas implicaciones de la evidencia neurocientífica en el contexto de los enfoques simulacionistas, teoricistas y modularistas en TdM.

$\mathrm{Al}$ reconocer que somos capaces de reproducir en nosotros mismos los patrones sensorio-motores característicos de las experiencias de otras personas, se establece que algunas ideas del simulacionismo estuvieron bien orientadas desde un principio, pues, aunque ignorando el verdadero mecanismo cerebral subyacente, las nociones generales de los simulacionistas se acercaron notablemente a lo que hoy aparece como un elemento funcional importante de un nivel básico de nuestra cognición social.

Por otro lado, como ha expuesto Riva (2006), es evidente que el SNE no es suficiente para explicar los niveles de cognición social más complejos que caracterizan a nuestra especie. En este sentido Becchio y Bertone (2003) exponen como ejemplo los casos de un juego como el ajedrez o de un deporte como el baloncesto, actividades sociales que pueden llegar a caracterizarse, no tanto por una interacción basada en la copia de movimientos sino por contraste, en la necesidad de ejecutar acciones distintas en procura de mantener la dinámica del comportamiento intencional colectivo.

Luego, aunque lo anterior podría propiciar que algunos mantengan su inclinación por acercamientos a la TdM de naturaleza teoricista, es necesario 
reconocer que la misma evidencia neurocientífica desafía el cimiento teoricista desde su mera base.

Las estructuras teóricas de los acercamientos teoricistas son edificadas con base en conceptos. El funcionamiento de la TdM del sujeto según estas aproximaciones, se relaciona directamente con la manipulación de ciertos conceptos conforme a reglas y objetivos (Carruthers \& Smith, 1996).

Los conceptos, desde estos enfoques, son vistos en términos de procesamiento de información abstracta, a saber, como simbolizaciones abstractas capaces de ser procesadas por nuestras mentes gracias a nuestra capacidad racional. Es decir, se trata de una noción de concepto típica en la psicología, la filosofía y la ciencia cognitiva a finales del siglo XX, época en que oportunamente se comenzaron a divulgar nuevas corrientes como la teoría neural del lenguaje o la cognición encarnada (Pecher \& Zwaan, 2005), tendencias precisamente dirigidas a una reformulación de la naturaleza de los conceptos, ahora entendidos en términos de percepción y acción, como representaciones elaboradas, recuperadas y mantenidas por la actividad particular de nuestro SNC y, en especial, por nuestros recursos de registro sensorio-motriz.

En palabras de Borghi (2005), cuando pensamos en un concepto lo que hacemos es recuperar una o varias grabaciones de la activación neuronal registrada durante algún episodio de nuestra percepción. Así entonces, desde esta perspectiva, tomando en cuenta la dinámica de la percepción y comprensión de las emociones según el funcionamiento asociado al SNE, y dada la naturaleza sensorio-motriz de los conceptos, no habría forma alguna de escindir las propuestas teoricistas de las propuestas simulacionistas. Los conceptos implicados en las estructuras teoricistas, y especialmente aquellos relacionados con los estados emocionales (propios y ajenos), serían -al menos parcialmente- estructurados y regulados por vía de nuestras capacidades sensorio-motoras espejo, donde eventos del tipo simulacionista jugarían un rol primordial.

Desde la evidencia del funcionamiento del SNE, las fronteras entre unas y otras aproximaciones vuelven a demostrarse como convenciones sumamente inapropiadas hacia el establecimiento de un constructo adecuado de la TdM.

Finalmente, los alcances del hallazgo del SNE también deben prolongarse hacia otro enfoque popular de la TdM: la aproximación modularista. El descubrimiento del SNE debe ser entendido por los entusiastas de las propuestas modularistas, como un desafío de primera prioridad en su construcción de esquemas de procesamiento de información en cognición social.

Es evidente que, de momento, ninguno de los esquemas de procesamiento de información enunciados en las propuestas modularistas (Scholl \& Leslie, 2001; Schneider, Schumann-Hengsteler \& Sodian, 2005; 
Seagal, 1996) parece capaz dar cuenta de la complejidad del accionar del SNE. Las propiedades multimodales que se observan en el SNE, donde cada neurona se desenvuelve como parte de sistemas sensoriales y motores de manera simultánea, y donde cada una varía su activación según la secuencia y activación en el tiempo, de algunos vecindarios neuronales próximos y otros más distales (Iacoboni, Moinar-Szakacs, Gallese, Buccino, Mazziotta \& Rizzolatti, 2005), son apenas algunas de las características funcionales básicas que los modularistas deberán intentar incorporar en sus modelos.

El reto para el modularismo quizá exija también una nueva revisión -o abandono- de su noción de módulo, en busca de un constructo que les permita acercarse a la caracterización fisiológica del SNE, y por ende, a un estilo de procesamiento del SNC que se sugiere importante para la TdM. Se requiere un esquema -sea modular o no- que pueda sostener la simultaneidad, flexibilidad, secuencialidad y multimodalidad de los subconjuntos de neuronas implicadas en el SNE.

La evidencia neurocientífica no llama a conciliar fragmentos de simulacionismo, teoricismo y modularismo como una ruta apropiada hacia una mejor perspectiva de la TdM. En este caso, desde la evidencia más bien se puede sugerir una reformulación necesaria para la TdM humana, ahora en términos de nuestra constitución biológica y de los recursos que en esa constitución pueden verse coaccionados.

La TdM debe ser entendida en el contexto del cuerpo. Debemos asumir que las características de este proceso cognitivo superior se encuentran ligadas a la constitución de nuestro SNC. Los alcances y limitaciones de nuestra TdM van de la mano con las modalidades funcionales de nuestro sistema nervioso, y el funcionamiento en espejo debe ser entendido como una de esas modalidades.

Que las funciones más importantes del SNE puedan verse implicadas apenas en el nivel más básico de nuestra TdM, debe entenderse como un aspecto altamente conveniente para nuestros propósitos. Desde ellas obtenemos un cimiento sólido a partir del cual emprender la exploración de otras modalidades y recursos que, más tarde, puedan ayudar a comprender niveles más complejos de nuestra cognición social.

\section{Referencias}

Adolphs, R. (2003). Cognitive neuroscience of human social behavior. Nature Reviews Neuroscience 4, 165-178.

Arce, M. (2005). Las huellas del zapatero. San José, Costa Rica: Alambique.

Baldissera, F., Cavallari, P., Craighero, L. \& Fadiga, L. (2001). Modulation of spinal excitability during observation of hand actions in humans. European Journal of Neuroscience 13, 190194. 
Baron-Cohen, S. (1997). Mindblindness: An essay on autism and theory of mind. Massachusetts: MIT Press.

Becchio, C. \& Bertone, C. (2003). Wittgenstein running: Neural mechanisms of collective intentionallity and we-mode. Consciousness and Cognition 13, 123-133.

Borghi, A. (2005). Object concepts and action. En D. Peacher, \& R. Zwann, (Eds). Grounding cognition: The role of perception and action in memory, language and thinking (pp. 8-34). Cambridge University Press.

Carruthers, P. \& Smith, P. (1996). Theories of theories of mind. Cambridge University Press.

Cocude, M., Mellet, E. \& Michel, D. (1999). Visual and mental exploration of visuo-spatial configurations: Behavioral and neuroimaging approaches. Psychological Research 62, 93107.

Cummings, R. (2002). Representation and unexploited content. En G. McDonald (Ed.). Teleosemantics (pp. 195-207). Oxford University Press.

Dennet, D. (1978). Beliefs about beliefs. Behavior and Brain Sciences 4, 568-570.

Di Pellegrino, G., Fadiga, L., Gallese, V. \& Rizzolatti, G. (1992). Understanding motor events: A neurophysiological study. Experimental Brain Research 91, 176-180.

Dominey, P. (2005). Constructions underlying theory of mind and language [en línea]. Disponible en: http://www.interdisciplines.org/coevolution/papers/11 [2005, $10 \mathrm{de}$ junio]

Fadiga, L., Fogassi, L., Pavesi, G. \& Rizzolatti, G. (1995). Motor facilitation during action observation: A magnetic stimulation study. Journal of Neurophysiology 72, 2608-2611.

Gazzaniga, M. (1999). El pasado de la mente. Barcelona: Andrés Bello.

Gazzaniga, M. (2005). Personal identity, neuroetbics and buman brain. Princeton Public Lectures. Princeton University.

Gallese, V., Fadiga, L., Fogassi, L. \& Rizzolatti, G. (1996). Action recognition in the premotor cortex. Brain 119, 593-609.

Gallese, V. \& Goldman, A. (1998). Mirror neurons and the simulation theory of mind-reading. Trends in Cognitive Sciences 12, 493-501.

Gallese, V. (2001). The 'shared manifold' hypothesis: From mirror neurons to empathy. Journal of Consciosness Studies 8, 33-35.

Gallese, V., Keysers, C. \& Rizzolatti, G. (2004). A unifying view of the basis of social cognition. Trends in Cognitive Science 8, 396-403.

Gallese, V. \& Lakoff, G. (2005). The brain's concepts: The role of the sensory-motor system in conceptual knowledge. Cognitive neuropsychology 21, 5-30.

Gallese, V. (2006). Intentional attunement: A neurophysiological perspective on social cognition and its disruption in autism. Brain Research 1079, 15-24.

Gentilucci, M., Fogassi, L., Luppino G., Matelli, M., Camarda, R. \& Rizzolatti, G. (1988). Functional organization of inferior area 6 in the macaque monkey. Experimental Brain Research 71, 475-490.

Gordon, R. (1996). 'Radical' Simulationism. En P. Carruthers (Ed.). Theories of theories of mind (pp. 11-21). Cambridge: Cambridge University Press.

Gordon, R. (1999). Simulation vs. theory-theory. En F. Wilson, \& C. Klein (Eds.). Enciclopedia of Cognitive Science. Massachusetts: MIT Press.

Gopnik, A. (1999). Theory of mind. En F. Wilson, \& C. Klein (Eds.). MIT. Enciclopedia of Cognitive Science. Massachusetts: MIT Press.

Heal, J. (1996). Simulation, theory and content. En P. Carruthers (Ed.). Theories of theories of mind (pp 11-21). Cambridge: Cambridge University Press.

Hobson, R. (1984). Early childhood autism and the question of egocentrism. Journal of Autism and Developmental Disorders 14, 85-104. 
Iacoboni, M., Moinar-Szakacs, I., Gallese, V., Buccino, G., Mazziotta, J. \& Rizzolatti, G. (2005). Grasping the intentions of others with one's own mirror neuron system. Plos Biology 3, 529-535.

Jackson, P., Meltzoff, A. y Decety, J. (2004). How do we perceive the pain of others? A window into the neural processes involved in empathy. Neuroimage 24, 771-779.

Jeannerod, M. (en prensa). Consciousness of action and self-consciousness: A cognitive neuroscience approach. En J. Roessler (Ed.). Agency and self awareness. Oxford University Press.

Peacher, D. \& Zwann, R. (2005). Grounding cognition: The role of perception and action in memory, language and thinking. Cambridge University Press.

Perner, J. (1996). Simulation as explicitation of predication-implicit knowledge about the mind: Arguments for a simulation-theory mix. En C.Carruthers, (Ed.). Theories of theories of mind (pp. 90-104). Cambridge University Press.

Perner, J. \& Kuhberger, S. (2003). Putting philosophy to work by making simulation theory testable: The case of endowment. En Ch. Kanzian, (Ed.). Persons. An interdisciplinary approach (pp. 33-44). Wien Press.

Premack, D. \& Woodruff, G. (1978). Does the chimpanzee have a theory of mind? Behavioral and Brain Sciences 2002, 532-535.

Riva, G. (2006). Being-in-the-world-with: Presence meets social and cognitive neuroscience. En G. Riva, M. Anguera, B.Wiederhold, \& F. Mantovani, (Eds.). From communication to presence: Cognition, emotions and culture towards the ultimate communicative experience (pp. 47-82). IOS Press.

Rizzolatti, G., Camarda, R., Fogassi L., Gentilucci, M., Luppino G. \& Matelli, M. (1988). Functional organization of inferior area 6 in the macaque monkey. Experimental Brain Research 71, 491-507.

Rizzolatti, G \& Arbib, M. (1998). Language within our grasp. Trends in Neuroscience 21, 188194.

Rizzolatti, G., Fogassi, L, \& Gallese, V. (2000). Cortical mechanisms subserving object grasping and action recognition: A new view on the cortical motor functions. En M. Gazzaniga, (Ed.). The Cognitive Neurosciences. Segunda edición. Massachusetts: MIT Press.

Rizzolatti, G. \& Craighero L. (2004). The mirror-neuron system. Annual Review of Neuroscience 27, 169-192.

Ruby, P. \& Decety, J. (2003). What you believe versus what you think they believe: A neuroimaging study of conceptual perspective-taking. European Journal of Neuroscience 17, 2475-2480.

Schneider, W., Schumann-Hengsteler, R. y Sodian, B. (2005) Young children's cognitive development: Interrelationships among executive functioning, working memory, verbal ability and theory of mind. New Jersey: Erlbaum.

Scholl, B. \& Leslie, A. (2001). Minds, modules and meta-analysis. Child Development 72, 696701.

Segal, G. (1996). The modularity of theory of mind. En P.Carruthers, y P. Smith (Eds.). Theories of theories of mind (pp. 141-157). Cambridge University Press.

Stich, S., Nichols, S., Leslie, A. \& Klein, D. (1996). Varieties of off-line simulation. En Carruthers, P. y Smith, P. (Eds). Theories of theories of mind (pp. 39-74). Cambridge University Press.

Tomasello, M. (1999). The cultural origins of human cognition. Harvard University Press.

Umiltá, M., Kohler, E., Gallese, V., Fogassi, L., Fadiga, L., Keysers, C. \& Rizzolatti, G. (2001). I know what you are doing: A neurophysiological study. Neuron 31, 155-165.

Weiskopf, D. (2002). Mental mirroring as the origin of attributions [en línea]. Florida: University of South Florida. Disponible en: luna.cas.usf.edu/ $\sim$ weiskopf/ papers/mental-mirroring.pdf [2005, 28 de mayo]. 
Wicker, B., Keysers, C., Plailly, J., Royet, J., Gallese, V. \& Rizzolatti, G. (2003). Both of us disgusted in my insula: The common neural basis of seeing and feeling disgust. Neuron 40, 655-664.

Wittgenstein, L. (1936). Investigaciones filosóficas. Barcelona: Crítica.

Recibido: 30 de octubre de 2005

Aceptado: 14 de junio de 2006 
